\title{
Performance Analysis and Comparison of Optical IDMA and Optical CDMA Techniques Using Unipolar Transmission Scheme
}

\author{
Ahmed M. Morsy and Eslam A. El-fiky \\ Electrical Engineering Department \\ Faculty of Engineering \\ Alexandria University \\ Alexandria 21544, Egypt \\ Email: ahmed_e_morsy@yahoo.com \\ Email: eslam_a_fiky@yahoo.com
}

\author{
Haitham S. Khallaf and Hossam M. H. Shalaby \\ Department of Electronics and \\ Communications Engineering \\ Egypt-Japan University of Science and Technology \\ Alexandria 21934, Egypt \\ Email: Eng.h.khallaf@gmail.com \\ Email: shalaby@ieee.org
}

\begin{abstract}
Interleave-division multiple-access (IDMA) has been recently proposed as a new spread spectrum multiple-access technique in optical communication systems. However, IDMA has not been studied for intensity modulation/direct detection (IM/DD) optical networks. In this paper, we introduce modifications to traditional IDMA decoding algorithm so as to make it suitable for IM/DD optical communication systems. In addition, we compare the performance, in terms of the bit-error rate (BER), of optical IDMA systems to the corresponding systems adopting optical CDMA. Our results reveal that under the above modifications, optical IDMA systems show significant improvement in the BER performance when compared to that of optical CDMA systems under same conditions
\end{abstract}

Index Terms-Optical IDMA, Optical CDMA, BER, Iterative decoding.

\section{INTRODUCTION}

In the last few years, there have been many endeavors to apply wireless multiple access techniques, such as code-division multiple-access (CDMA) and orthogonal frequency-division multiple-access (OFDMA), in optical communications as well [1],[2]. Many of these endeavors, especially optical CDMA (OCDMA), showed good performance in the optical domain as well [1].

Recently, wireless interleave-division multiple-access (IDMA) has been introduced as a new multiple access spread spectrum technique [3]-[5], that is similar to CDMA. However, IDMA uses user specific interleavers to separate different users instead of using user specific codes as in CDMA. Also, IDMA allows the use of a low complexity iterative multiuser detector [6]-[8]. Performance comparison of wireless IDMA and CDMA has shown that IDMA performs better than CDMA [9]-[10].

Optical IDMA (OIDMA) has been introduced in [11] and has shown a good performance for a high number of users. The authors in [11] have simulated an OIDMA system over a nonlinear optical channel. However, they just used the decoding algorithm proposed in [9] which is not realistic for the noncoherent IM/DD optical communication systems, where a unipolar transmission scheme should be used. In this paper, we modify the decoding algorithm to be suitable for the unipolar transmission case, specifically, on-off-keying (OOK) modulation scheme. In addition, we introduce the chip-bychip decoding algorithm used in wireless CDMA systems [9] to the unipolar OCDMA systems under our new modifications. Finally, we provide a performance comparison of both OIDMA and the chip-by-chip decoded OCDMA systems. Our results reveal that OIDMA has better BER performance than OCDMA, allowing more number of users to be accommodated in the optical channel.

The rest of the paper is organized as follows. In Section II, we introduce the system model. Section III, is devoted for the simulation results. Finally, the conclusion is given in Section IV.

\section{System MODEL}

We consider a multiuser optical communication system with $\mathrm{K}$ users. The system model for OIDMA is shown in Fig. 1.

\section{A. Transmitter side}

The upper part of Fig. 1 shows the OIDMA transmitter with $K$ simultaneous users. The information bits for user $k \in\{1,2, \ldots, K\}$, denoted by $\left\{b_{i}^{(k)} \in\{0,1\}, i=1, \ldots, N_{b}\right\}$ where $N_{b}$ is the information block length of user $k$, are spread using common low rate spreading codes of length $C$. This generates a coded sequence $\left\{c_{j}^{(k)}, j=1,2, \ldots, N_{c}\right\}$ where $N_{c}=$ $N_{b} C$. Next, a chip level user specific interleaver $\pi^{(k)}$ is applied to produce the transmitted sequence $\left\{x_{j}^{(k)}, j=1,2, \ldots, N_{c}\right\}$. The interleavers are generated randomly and they are the only means to separate the data of users in OIDMA. Removing the user specific interleavers $\pi^{(k)}$ and using user specific spreading codes $\left\{s^{(k)} \in\{0,1\}, k=1,2, \ldots, K\right\}$ for user separation leads to OCDMA structure. In order to apply the unipolar spreading sequences to optical CDMA communications, sequence inversion keyed (SIK) system is employed as proposed 


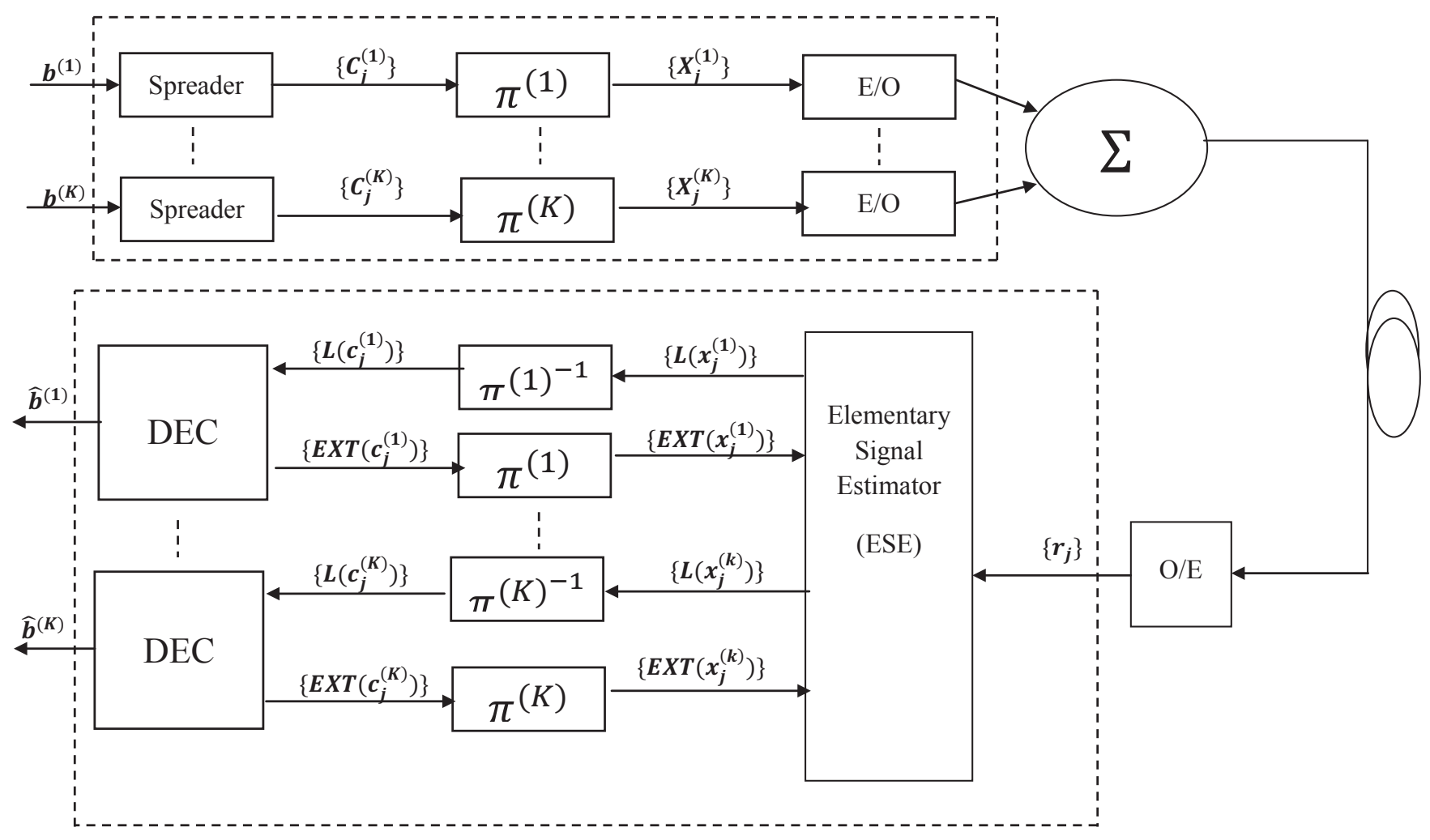

Fig. 1. OIDMA transmiter and reciever architecture.

in [14],[15]. Electrical to optical converter $(E / O)$ is used to generate the optical pulses.

\section{B. Channel Model}

Pulse propagation in the optical fiber is modeled using the normalized non-linear Schrödinger (NLS) equation [12]

$$
\frac{\delta U}{\delta \xi}=\frac{i s}{2} \frac{\delta^{2} U}{\delta \tau^{2}}+i N^{2}|U|^{2} U
$$

where $s=\operatorname{sgn}\left(\beta_{2}\right)= \pm 1$, depending on whether fiber dispersion is normal $(s=1)$ or anomalous $(s=-1)$. $U$ is the normalized amplitude given by

$$
U=\frac{A}{\sqrt{P_{0}}}
$$

where $A, P_{0}$ and $\beta_{2}$ are the pulse envelope, the peak power of the incident pulse, and the second order dispersion parameter respectively. $\xi$ is the normalized distance, given by

$$
\xi=\frac{z}{L_{D}}
$$

where $z$ is the distance and $L_{D}$ is the dispersion length, given by

$$
L_{D}=\frac{T_{0}^{2}}{\left|\beta_{2}\right|}
$$

where $T_{0}$ is the initial pulse width. $\tau$ is the normalized time, given by

$$
\tau=\frac{T}{T_{0}}
$$

$N$ is a parameter that gives an indication to the relative effect of group velocity dispersion (GVD) and self phase modulation (SPM) on pulse shape as it propagates through the fiber. GVD is more dominant for $N$ much less than 1 while SPM is more dominant for $N$ much greater than 1 . Both have the same effect on pulse shape for $N=1$, and $N$ is given by [12]

$$
N=\sqrt{\frac{L_{D}}{L_{N L}}}=\sqrt{\frac{\gamma P_{0} T_{0}^{2}}{\left|\beta_{2}\right|}}
$$

where $L_{N L}$ is the nonlinear length

$$
L_{N L}=\frac{1}{\gamma P_{0}}
$$

and $\gamma$ is the non-linear parameter.

In this paper, we use split step Fourier method (SSFM) to find a numerical solution for the NLS equation [12]. In SSFM the fiber is divided into small segments of equal length $w$. For each segment, the effects of dispersion and non-linearity are considered independently as shown in Fig. 2. 


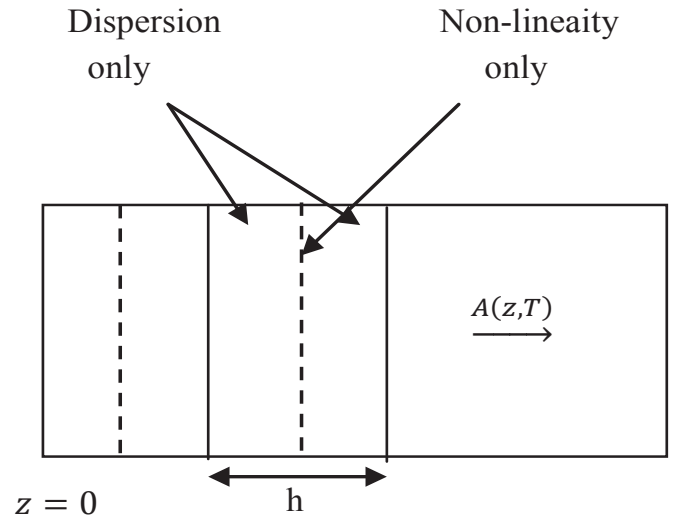

Fig. 2. Schematic explanation of the split step fourier method.

\section{OIDMA Receiver}

The optical IDMA receiver, shown in Fig. 1, is an iterative receiver that utilizes the soft decoding (turbo) algorithm [6],[9]. At the front end of the receiver, we use an optical detector that converts the received light into electrical current according to

$$
I=R P
$$

where $R$ is the responsivity of the photodetector, $I$ is the output electric current and $P$ is the received optical power.

The rest of the receiver architecture is the same as that used in wireless IDMA [3]-[5] with modifications to the decoding algorithm to fit with the OOK transmission used in optical communications. The iterative chip-by-chip receiver in the lower part of Fig. 1 consists of an elementary signal estimator (ESE) and a bank of $K$ single-user a posteriori probability decoders for the despreading operation (DEC) working in a turbo-type manner. In the wireless IDMA system, considering BPSK, the received symbols $r_{j}$ are given by [9]

$$
r_{j}=\sum_{k=1}^{K} h^{(k)} x_{j}^{(k)}+n_{j}
$$

where $x_{j}^{(k)} \in\{+1,-1\}$ is the transmitted symbol from user $k$ at time instant $j, h^{(k)}$ is the channel coefficient for user $k$ assuming flat fading channel and $n_{j}$ is an additive white Gaussian noise (AWGN).

In intensity-modulation/direct-detection optical communication systems, negative symbols cannot be used. As a result, we use OOK modulation for the transmitted signal. In this system the received signal remains the same as in (9), with $x_{j}^{(k)} \in\{+1,0\}$. For the chip-by-chip decoding algorithm proposed in [9] we concentrate on each user's chip $x_{j}^{(k)}$ separately. Thus, (9) should be modified to

$$
r_{j}=h^{(k)} x_{j}^{(k)}+\zeta_{j}^{(k)}
$$

where $\zeta_{j}^{(k)}=r_{j}-h^{(k)} x_{j}^{(k)}$ represents the noise plus multiple access interference term with respect to $x_{j}^{(k)}$. From (9), the mean and variance of $r_{j}$ can be obtained as

$$
E\left(r_{j}\right)=\sum_{k=1}^{K} h^{(k)} E\left(x_{j}^{(k)}\right)
$$

and

$$
\operatorname{var}\left(r_{j}\right)=\sum_{k=1}^{K}\left|h^{(k)}\right|^{2} \operatorname{var}\left(x_{j}^{(k)}\right)+\sigma^{2}
$$

respectively. Unlike [9], since we are using OOK, $E\left(x_{j}^{(k)}\right)$ and $\operatorname{var}\left(x_{j}^{(k)}\right)$ are initialized to be 0.5 and 0.25 , respectively, and then modified after each decoder iteration.

Using the central limit theorem for large number of users, $\zeta_{j}^{(k)}$ in $(10)$ can be approximated by a Gaussian random variable with

$$
\begin{gathered}
E\left(\zeta_{j}^{(k)}\right)=E\left(r_{j}\right)-h^{(k)} E\left(x_{j}^{(k)}\right) \\
\operatorname{var}\left(\zeta_{j}^{(k)}\right)=\operatorname{var}\left(r_{j}\right)-\left|h^{(k)}\right|^{2} \operatorname{var}\left(x_{j}^{(k)}\right)
\end{gathered}
$$

The ESE outputs are the logarithm likelihood ratios (LLRs) of $x_{j}^{(k)}$ computed as

$$
\begin{aligned}
L\left(x_{j}^{(k)}\right) & =\log \frac{\operatorname{Pr}\left(x_{j}^{(k)}=+1 \mid r_{j}\right)}{\operatorname{Pr}\left(x_{j}^{(k)}=0 \mid r_{j}\right)} \\
& =\log \frac{\exp \left(-\frac{\left(r_{j}-E\left(\zeta_{j}^{(k)}\right)-h^{(k)}\right)^{2}}{2 \operatorname{var}\left(\zeta_{j}^{(k)}\right)}\right)}{\exp \left(-\frac{\left(r_{j}-E\left(\zeta_{j}^{(k)}\right)\right)^{2}}{2 \operatorname{var}\left(\zeta_{j}^{(k)}\right)}\right)} \\
& =\frac{2 h^{k}\left[\left(r_{j}-E\left(\zeta_{j}^{(k)}\right)\right)-\left|h^{(k)}\right|^{2}\right]}{2 \operatorname{var}\left(\zeta_{j}^{(k)}\right)}
\end{aligned}
$$

For user- $k$, the corresponding ESE outputs $\left\{L\left(x_{j}^{(k)}\right), j=\right.$ $1,2, \ldots, J\}$ are de-interleaved to form $\left\{L\left(c_{j}^{(k)}\right), j=\right.$ $1,2, \ldots, J\}$ and delivered to the DEC for user- $k$. The DEC performs a soft-in/soft-out chip-by-chip de-spreading operation as follows [9]

$$
L\left(d_{1}^{(k)}\right)=\sum_{j=1}^{S} S_{j}^{(k)} L\left(c_{j}^{(k)}\right)
$$

where $\left\{S_{j}^{(k)}, j=1,2, \ldots, J\right\}$ is the spreading sequence of user $k$ such that $S_{j}^{(k)} \in\{1,0\}$. The extrinsic LLR for a chip $c_{j}^{(k)}$ is given by

$$
\operatorname{Ext}\left(c_{j}^{(k)}\right)=S_{j}^{(k)} L\left(d_{1}^{(k)}\right)-L\left(c_{j}^{(k)}\right)
$$

The extrinsic LLRs form the outputs of the DEC are fed back to the ESE after interleaving. In the next iteration, $\operatorname{Ext}\left(x_{j}^{(k)}\right)$ are used to update $E\left(x_{j}^{(k)}\right)$ and $\operatorname{Var}\left(x_{j}^{(k)}\right)$ as 


$$
\begin{aligned}
E\left(x_{j}^{(k)}\right) & =\operatorname{Pr}\left(x_{j}^{(k)}=1\right)(1)+\operatorname{Pr}\left(x_{j}^{(k)}=0\right)(0) \\
& =\frac{\operatorname{Pr}\left(x_{j}^{(k)}=1\right)}{\operatorname{Pr}\left(x_{j}^{(k)}=1\right)+\operatorname{Pr}\left(x_{j}^{(k)}=0\right)} \\
& =\frac{\exp \left(\operatorname{Ext}\left(x_{j}^{(k)}\right)\right)}{\exp \left(\operatorname{Ext}\left(x_{j}^{(k)}\right)\right)+1}
\end{aligned}
$$

$$
\begin{aligned}
\operatorname{var}\left(x_{j}^{(k)}\right) & =E\left(x_{j}^{(k)^{2}}\right)-E\left(x_{j}^{(k)}\right)^{2} \\
& =\operatorname{Pr}\left(x_{j}^{(k)}=1\right)(1)^{2}-E\left(x_{j}^{(k)}\right)^{2} \\
& =E\left(x_{j}^{(k)}\right)-E\left(x_{j}^{(k)}\right)^{2}
\end{aligned}
$$

This iterative process is repeated for a predetermined number of iterations. After the final iteration, the data $\operatorname{LLR} L\left(d_{1}^{(k)}\right)$ is hard limited to produce the estimate data sequence. The detection algorithm in IDMA does not rely on coding unlike other methods [3]-[9], but introducing coding can further enhance performance (details omitted here).

\section{CDMA Receiver}

For the comparison, to be fair, we use an iterative chipby-chip decoder for the CDMA as well. An elementary signal estimator (ESE) block first calculates the LLRs of the received chips. The output LLRs are then correlated with the bipolar version of the spreading code [13]-[15] producing the despreaded LLR $L\left(y_{j}^{(k)}\right)$. The extrinsic LLR is then computed by

$$
\operatorname{Ext}\left(c_{j}^{(k)}\right)=L\left(y_{j}^{(k)}\right)-L\left(c_{j}^{(k)}\right)
$$

The extrinsic LLRs form the outputs of the DEC, and are fed back to the ESE after spreading again using the bipolar form of the spreading code. In the next iteration, $\operatorname{Ext}\left(x_{j}^{(k)}\right)$ are used to update $E\left(x_{j}^{(k)}\right)$ and $\operatorname{var}\left(x_{j}^{(k)}\right)$ in the same way as in IDMA.

\section{Simulation Results}

In this section, we provide a Matlab simulation for the biterror rate (BER) of both OIDMA and OCDMA systems. Fig. 3 shows a BER comparison for both OIDMA and OCDMA systems with different numbers of simultaneous users. Also, Fig. 4 shows a similar comparison but with different number of the decoding iterations for both OIDMA and OCDMA systems.

For our simulation, the information block length is set to be 256 bits, OOK is used for bit representation at the transmitter side, and the spreading code length is set to 64 . We also consider a single mode fiber (SMF) operating at $1.55 \mu \mathrm{m}$ wavelength with normal dispersion $(s=1)$ and the nonlinear parameter $N$ is set to 1 . Also, the input to the optical fiber is chosen to be a Gaussian pulse [12]. Both thermal and shot noise are considered at the reciever. The responsivity and the

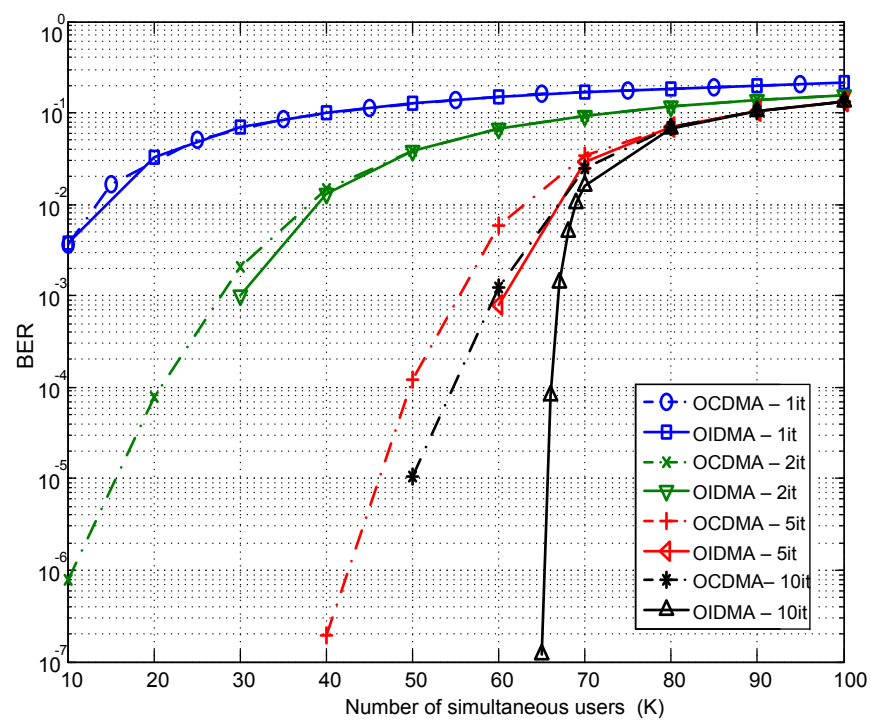

Fig. 3. BER of OIDMA and OCDMA systems versus number of users for 10 decoding iterations.

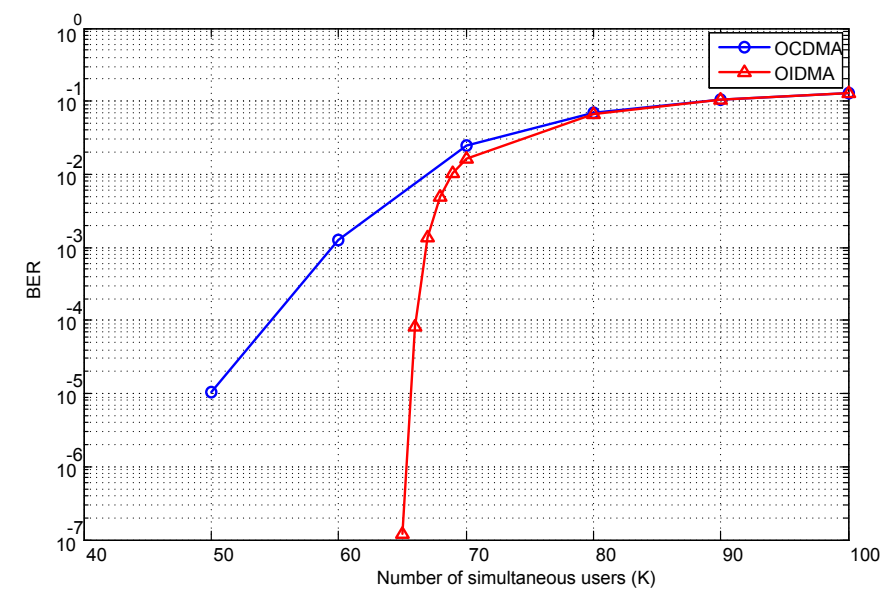

Fig. 4. BER of OIDMA and OCDMA versus number of users for 1, 2, 5 and 10 decoding iterations.

maximum bit rate are taken as 0.6 and $1 \mathrm{Gbps}$, respectively. For IDMA, we use random interleavers to separate different users and alternating $\{1,0,1,0,1, \ldots\}$ spreading sequence for all users. In contrary, for CDMA, we totally remove the interleavers and use random spreading sequences for user separation. Since the IDMA decoder used here is designed only for flat fading channel, we have compensated for the effect of the dispersion in the optical fiber before the iterative decoder.

It is observed from Fig. 3 that OIDMA significantly improves the number of users to be accommodated in the same channel. For example, at BER $=10^{-5}$, OIDMA can accommodate about 65 users, while OCDMA can only accommodate about 50 users. That is an increase of about 30\% in the number of users is obtained when using OIDMA technique. In addition this ratio increases as the required BER decreases, 
where the number of users that can be accommodated when using OCDMA decreases dramatically, while that when using OIDMA remains almost constant. However, for higher values of BER (more than $10^{-2}$ ), both OIDMA and OCDMA systems can accommodate similar number of users. This is because as the number of users exceeds the spreading code length, the cross correlation between the user specific interleavers dramatically increases [16]. This case of small BER is in fact not of practical importance for optical systems.

Figure 4 shows the BER of both OIDMA and OCDMA systems versus the number of users for different values of decoding iterations. It can be seen from the figure that as the number of decoding iterations increases, the BER performance improves. However, the amount of improvement decreases as the number of decoding iterations increases, meaning that there exists a saturation number of iterations after which, there is no more improvement. It is also clear from the figure that for one decoding iteration, both OIDMA and OCDMA give identical BER curves. However, as the number of iterations increases, the difference between the two curves also increases in the favor of OIDMA.

\section{CONCLUSION}

The performance of OIDMA communication systems, in terms of BERs, has been presented using computer simulation. In our simulation, the traditional wireless IDMA decoding algorithm has been modified to fit the noncoherent IM/DD optical communication systems. Furthermore, the BER performance has been compared to that of the chip-by-chip decoded OCDMA systems. It has been shown that for a given value of BER, OIDMA system can accommodate more users than that of OCDMA. In fact for BER values of interest (less than $10^{-3}$ ), this improvement is more than $30 \%$. In addition, it has been shown that, for one decoding iteration, both OIDMA and OCDMA give identical BER curves. However, as the number of iterations increases, the difference between the two curves also increases in the favor of OIDMA.

\section{REFERENCES}

[1] J. A. Salehi, "Code division multiple access techniques in optical fiber networksart I: Fundamental principles," IEEE Trans. Commun., vol.37, pp. 82433, Aug. 1989.

[2] J.Armstrong, "OFDM for optical communications," J. Lightw. Technol., vol. 27, no.3, 2009.

[3] Li Ping, L. Liu, K. Y. Wu, and W. K. Leung, "Interleave-division multipleaccess (IDMA) communications,"in Proc. 3rd International Symposium on Turbo Codes and Related Topics, 2003, pp. 17380.

[4] Li Ping, L. Liu, K. Y. Wu, and W. K. Leung, "Interleave-division multipleaccess," IEEE Transactions on Wireless Communications, vol. 5, no. 4,pp. 93847, April 2006.

[5] L. Liu, W. K. Leung, and Li Ping, "Simple chip-by-chip multi-user detection for CDMA systems," in Proc. IEEE VTC003-Spring, Jeju,Korea, Apr. 2003, pp. 2157161.
[6] C. Bmou and A. Glavieux. "Near Shannon limit error correcting coding and decoding: Turbo codes," IEEE Trans. Commun., vol. 44, pp. 12611271. Oct. 1996.

[7] X. Wang and H. V. Poor, "Iterative (turbo) soft interference cancellation and decoding for coded CDMA.” IEEE Trans. Commun.,vol. 47. pp.10461061.July 1999.

[8] R. H. Mahadevappa and J. G. Raakis, "Mitigating multiple access interference and intersymbol interference in uncoded CDMA Systems with chip-level interleaving." IEEE Trans Wireless Commun..vol. 1, pp.781792, Oct. 2002.

[9] W. K. Leung, Lihai Liu and Li Ping, "Interleaving-Based Multiple access and iterative chip-by-chip multiuser detection," IEICE Trans. on Commun., vol. E86-B, pp. 3634-3637, Dec. 2003.

[10] X. Liu, X. Xiong, and P. Yang, "Performance of IDMA and CDMA Systems Based on Simulink, "Wireless Communications Networking and Mobile Computing (WiCOM), 2010.

[11] M. Shukla1, Monika Gupta, Shashi Tiwari, P.S. Sharma, Somendra Shukla, "Optical Interleave-Division Multiple-Access Scheme for Long Distance Optical Fiber Communication," 2010 IEEE International Conference on Computational Intelligence and Computing Research.

[12] G.Agrawal,"Non-linear Fiber Optics," fourth edition, Academic press 2007.

[13] M. J. Parham, C. Smythe, and B. L. Weiss, "Code Division Multiple-Access Techniques for Use in Optical-Fiber Local-Area Networks,'Electron and, commun.Eng. J., pp.20312, 1992.

[14] T. Oarrell and S. Lockmann, "Performance Analysis of an Optical correlator Receiver for SIK DS-CDMA Communication Systems," Electronics Letters, Vol.30, No.1, pp.635, 1994.

[15] Akio Tsuneda and Takashi Yoshida, "Performance Evaluation of AsynchronousDS/CDMA Communications Using Unipolar Codes,"in the 20th European Conference on Circuit Theory and Design (ECCTD), 2011.

[16] Ioachim Pupeza, Aleksandar Kavcic and Li Ping, "Efficient Generation of Interleavers for IDMA," IEEE ICC 2006 proceedings. 
This page is intentionally left blank. 